\title{
PREHEAT ISSUES IN HYDRODYNAMIC HEDLA EXPERIMENTS
}

\author{
C.C. KURANZ ${ }^{1}$, R.P. DRAKE ${ }^{1}$, K.K. DANNENBERG ${ }^{1}$, P.J. SUSALLA ${ }^{1}$, \\ D.J. KREMER ${ }^{1}$, T. BOEHLY ${ }^{2}$ and J. KNAUER ${ }^{2}$ \\ ${ }^{1}$ University of Michigan; E-mail: ckuranz@umich.edu \\ ${ }^{2}$ Laboratory for Laser Energetics
}

(Received 30 April 2004; accepted 9 June 2004)

\begin{abstract}
Hydrodynamic experiments have become a very active area within High Energy Density Laboratory Astrophysics. In such experiments, preheat of an interior surface due to heating prior to shock arrival can alter the initial conditions for further evolution and can change the nature of the experiment (Olson et al., 2003). Unfortunately, preheat cannot typically be detected without undertaking dedicated experiments for this purpose. We have designed such experiments, relevant to hydrodynamic instability experiments using Omega Laser at intensities of $\sim 10^{15} \mathrm{~W} / \mathrm{cm}^{2}$. Simulations using the HYADES code suggest that radiative preheat alone causes the interface to move approximately $2 \mu \mathrm{m}$ before the blast wave reaches it. Hot-electron preheat could cause much larger motions. These experiments will use VISAR to examine the motion of an aluminum sample layer at the rear interface of a standard hydrodynamic target during the period before the shock reaches it (Allen and Burton, 1993).
\end{abstract}

\section{Introduction and Target Design}

One of the major areas for experiments of interest to astrophysics using highenergy-density facilities is hydrodynamics. This is a natural connection, in that many astrophysical systems, and many high-energy-density ones, can be accurately described by the Euler equations (Ryutov et al., 1999). Experiments of this type have been done or are planned at numerous laser facilities (and at $\mathrm{Z}$ pinches). A partial list includes Nova (Drake et al., 2000; Kane et al., 2000; Remington et al., 1997), Omega (Drake et al., 2002; Robey et al., 2001), Gekko (Kang et al., 2001) and the National Ignition Facility. Such experiments typically seek to produce strong decelerations or strong shear flows, leading one to seek to produce the highest feasible velocities. The desire for strong acceleration leads one in turn to use the highest-feasible laser irradiance and to employ low-density materials. (Plastics have been common; foams have been discussed). Both of these directions lead one toward the regime in which preheat of the experimental system will have significant effects. Some exploration of this issue is the goal of the experiments discussed here.

Preheat occurs when energy is transported, either by radiation or by particles, deep into an experimental system from a region of large energy deposition. In the cases of interest here, a laser-irradiated surface can be a source both of 
$\mathrm{X}$-rays, produced in the hot $(\sim \mathrm{keV})$ coronal plasma, and of "hot" electrons, having suprathermal energies of tens of $\mathrm{keV}$ and produced in consequence of laser-plasma instabilities. Some of the hydrodynamic experiments produce a blast wave and use it to create Rayleigh-Taylor instabilities by shocking then decelerating an interface where the density drops. The interface is typically prepared by imposing a very specific initial condition. In such experiments, which are our specific concern here, preheat can modify the initial conditions before the blast wave arrives at the interface. Thus, an accurate understanding of such experiments must include an assessment of the preheat that is present.

This problem is complicated because such an assessment cannot be entirely computational. Current simulation codes, using multigroup diffusive radiation transport, are believed to accurately calculate the radiation preheat. However, they make no attempt to evaluate the hot-electron preheat. What is known is that the laser irradiances used in the experiments of interest, of order $10^{15} \mathrm{~W} / \mathrm{cm}^{2}$ at laser wavelengths of $0.35 \mu \mathrm{m}$, can correspond to significant production of hot electrons (Wolf Seka, Laboratory for Laser Energetics, private communication). This motivates the current experiments, the design of which is discussed here.

Figure 1 shows one of the target designs to be used for the preheat experiments. Ten beams of the Omega laser (Boehly et al., 1995) are incident from the left onto the polyimide surface. These produce a laser intensity of approximately $10^{15} \mathrm{~W} / \mathrm{cm}^{2}$ at laser wavelengths of $0.35 \mu \mathrm{m}$, using $1 \mathrm{~ns}$ flat-topped pulses, on a spot of $800 \mu \mathrm{m}$ FWHM. This configuration is identical to that used in numerous planar hydrodynamic experiments (Miles et al., 2003a,b; Robey et al., 2001, 2003). The $150-\mu \mathrm{m}$ thick polyimide (C22H10O5N2) layer would normally be followed by a lower-density material. In this case it is followed by an Al layer, $10 \mu \mathrm{m}$ thick here but $0.2 \mu \mathrm{m}$ in other cases. The $10 \mu \mathrm{m}$ thickness was chosen to absorb and respond to the anticipated preheat as is discussed below. Behind the Al layer is a window of $\mathrm{SiO}_{2}$, through which a Velocity Interferometer System for Any Reflector (VISAR) (Barker and Hollenback, 1972; Sheffield et al., 1986) can use a laser of $532 \mathrm{~nm}$ wavelength to interrogate the surface to see the motion induced by the preheat. The $\mathrm{SiO}_{2}$ layer was intended to tamp the aluminum surface, so that it would remain reflective even if heated to some degree. This approach has been used successfully

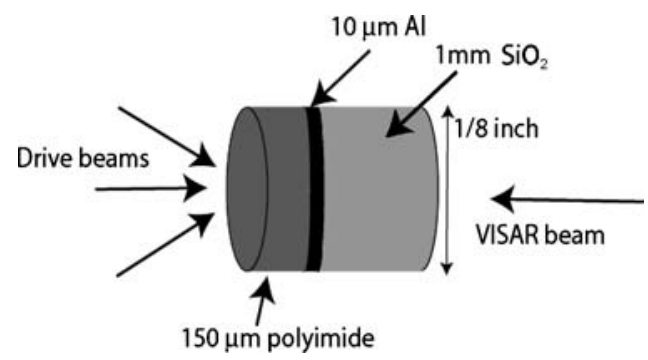

Figure 1. Schematic of one of the preheat targets. 
in experiments at Sandia National Laboratories. If there were so much preheat that the $\mathrm{SiO}_{2}$ became conductive, then the VISAR diagnostic would prove unable to make a measurement because of absorption. Part of the experiment is to see whether this occurs. If the preheat were radiative only and as predicted by the simulations, then the temperature in the $\mathrm{SiO}_{2}$ would remain below $0.3 \mathrm{eV}$ and one could hope to see the $\mathrm{Al}$ layer until the shock arrived. In addition, an optical pyrometer will be used to observe heating of the $\mathrm{Al}$ material by detecting the thermal emission from the target. The interior surface of the $\mathrm{SiO}_{2}$ window is coated by $0.2 \mu \mathrm{m}$ of $\mathrm{Al}$ to assure a high-quality signal for these diagnostics.

\section{Predictions from Simulations and Calculations}

The experimental system for the preheat experiment was simulated using HYADES (Larsen and Lane, 1994), a one-dimensional, Lagrangian, radiation-hydrodynamics code that is widely used for experimental scoping. It uses multigroup radiation transport, allowing it to predict the effects of the X-ray photons at the interface before the shock arrives. It is possible that hot-electron preheat will greatly increase the movement of the interface, as is discussed further below.

Hyades runs were performed with a $150 \mu \mathrm{m}$ layer of polyimide, $10 \mu \mathrm{m}$ of $\mathrm{Al}$, and $1 \mathrm{~mm}$ of $\mathrm{SiO}_{2}$. The output from these runs can be seen in Figures 2 and 3, where different lines indicate different times throughout the run. Figure 2 shows the velocity of the interface to be $\sim 1 \mu \mathrm{m} / \mathrm{ns}$ and the shock arriving at the interface at $\sim 2.2 \mathrm{~ns}$. Thus the interface moves $\sim 2.2 \mu \mathrm{m}$ before the shock. Figure $3 \mathrm{a}$ and $\mathrm{b}$ show the temperature of the interface to be $\sim 1 \mathrm{eV}$ and a change in the density, respectively, prior to shock arrival.

Since HYADES cannot account for hot electrons, calculations of the energy flux of hot electrons through the interface were made. The total energy deposited in the preheated material, on the assumption that the temperature is increased uniformly,

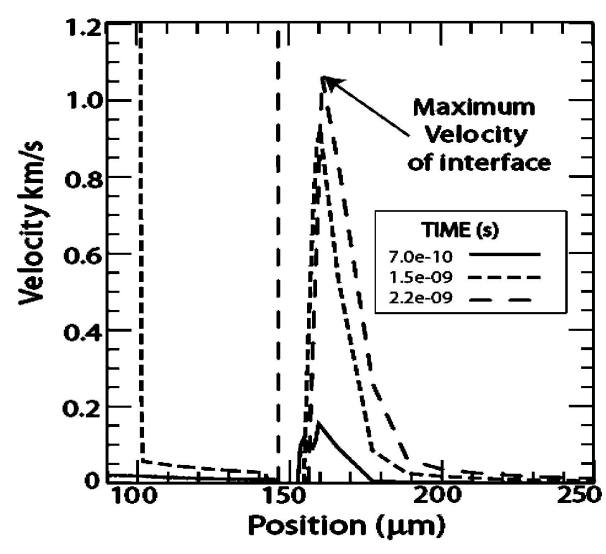

Figure 2. Velocity due to radiation preheat. 

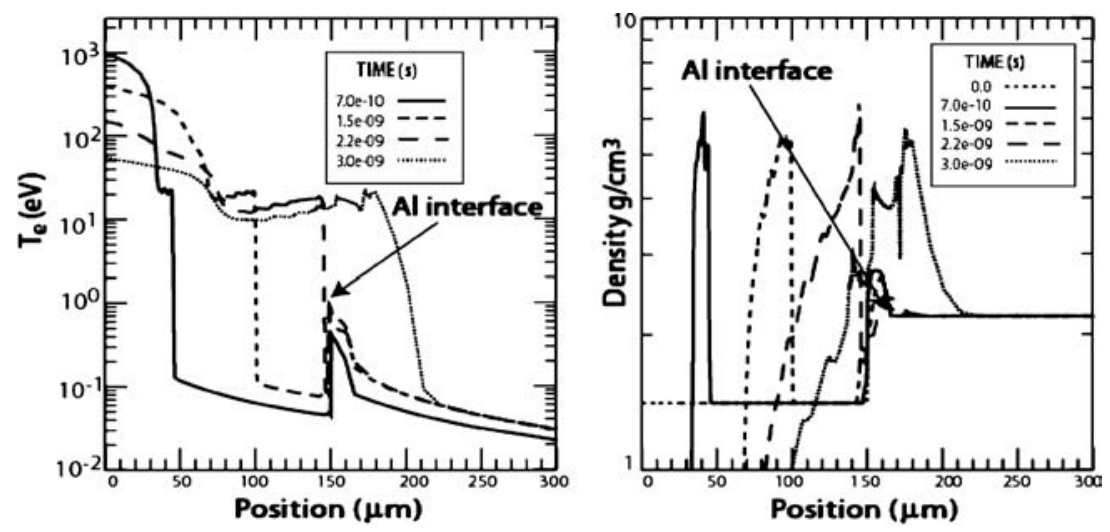

Figure 3. Temperature and density changes due to radiation preheat.

is given by $\left[\rho k_{B} T /\left(A m_{p}\right)\right] d a$, where $\rho$ is the density, $T$ is the temperature, $A$ is the atomic mass, $m_{p}$ is the mass of the proton, $d$ is the thickness, $a$ is the area of the laser spot, and $k_{B}$ is the Boltzmann constant. To estimate a preheat temperature, we assume that approximately $0.1 \%$ of the laser energy $(4 \mathrm{~J})$ goes into hot electrons that are deposited in the polyimide underneath the laser spot. (The total energy of the hot electrons produced would be a large multiple of this number, probably more than ten times larger but well within the range of plausibility for this experiment.) Then using $\rho=1.4 \mathrm{~g} / \mathrm{cm}^{3}$ for polyimide, $d=150 \mu \mathrm{m}$, and $a=0.5 \mathrm{~mm}^{2}$, one finds the preheat temperature to be approximately $5 \mathrm{eV}$. It is thus possible that the hot electrons cause a much larger motion than the radiative preheat alone. This motion would be expected to alter the structure on the surface and to affect the subsequent instability evolution.

\section{Conclusions}

Through simulations and calculations we have found that X-ray photons and hot electrons from the laser in a hydrodynamic experiment may cause preheat at an interface. This heats the interface and causes it to move prior to the arrival of the shock, changing the initial conditions of the experiment. We have designed an experiment to detect such preheat and to assess its magnitude. These experiments will soon be carried out in order to find out how much energy actually reaches the interface.

\section{Acknowledgments}

This work is supported by the U. S. Department of Energy under grants DE-FG0399DP00284 and DE-FG03-00SF22021. 


\section{References}

Allen, D.A. and Burton, M.G.: 1993, Nature 363, 54.

Barker, L.M. and Hollenback, R.E.: 1972, J. Appl. Phys. 43, 1669.

Boehly, T.R. et al.: 1995, Rev. Sci. Intsr. 66, 508.

Drake, R.P. et al.: 2000, Phys. Plasmas 7, 2142.

Drake, R.P. et al.: 2002, Astrophys. J. 564, 896.

Kane, J., Arnett, D., Remington, B.A., Glendinning, S.G., Bazan, G., Drake, R.P. and Fryxell, B.A.: 2000, Ap. J. Suppl. 127, 365.

Kang, Y.G. et al.: 2001, Plasma Phys. Rep. 27, 843.

Larsen, J.T. and Lane, S.M.: 1994, J. Quant. Spectrosc. Radiat. Transfer 51, 179.

Miles, A.R., Braun, D.G., Edwards, M.J., Robey, H.F., Drake, R.P. and Leibrandt, D.R.: 2003a, Phys. Plasmas, submitted.

Miles, A.R., Edwards, M.J. and Robey, H.F.: 2003b, in Inertial Fusion and Science Applications, The effect of a short-wavelength mode on the nonlinear evolution of a long-wavelength perturbation driven by a strong blast wave (Monterey, CA), submitted.

Olson, R.E., Leeper, R.J., Nobile, A. and Oertel, J.A.: 2003, Phys. Rev. Lett. 91.

Remington, B.A. et al.: 1997, Phys. Plasmas 4, 1994.

Robey, H.F. et al.: 2001, Phys. Plasmas 8, 2446.

Robey, H.F., Zhou, Y., Buckingham, A.C., Keiter, P., Remington, B.A. and Drake, R.P.: 2003, Phys. Plasmas 10, 614.

Ryutov, D.D., Drake, R.P., Kane, J., Liang, E., Remington, B.A. and Wood-Vasey, M.: 1999, Ap. J. 518, 821.

Sheffield, S.A., Rogers, J.W., Castaneda, J.N. and Gupta, V.M.: 1986, in Shock Waves in Condensed Matter (New York: Plenum), p. 541. 